\title{
Importance of the Keap1-Nrf2 pathway in NSCLC: Is it a possible biomarker? (Review)
}

\author{
RAÚL BARRERA-RODRÍGUEZ \\ Department of Biochemistry and Environmental Medicine, \\ National Institute of Respiratory Diseases, Mexico City 14080, Mexico
}

Received September 6, 2017; Accepted August 2, 2018

DOI: $10.3892 /$ br.2018.1143

\begin{abstract}
Worldwide, lung cancer remains the most common cause of cancer-related mortality, with non-small cell lung cancer (NSCLC) accounting for $85 \%$ of all diagnosed lung cancer cases. Chemotherapy is considered the standard of care for patients with advanced NSCLC; however, the tumors can develop mechanisms that inactivate these drugs. Comparative genomic analyses have revealed that disruptions in the kelch-like ECH-associated protein 1 (Keap1)-nuclear factor erythroid-2-related factor-2 (Nrf2) pathway are frequent in NSCLC, although Nrf2 mutations occur less frequently than Keap1 mutations. As the Keap1-Nrf2 pathway appears to be a primary regulator of key cellular processes that aid to resist the action of chemotherapy drugs, the clinical implementation of Nrf2 inhibitors in patients with advanced NSCLC may be a useful therapeutic approach for patients harboring KEAP1-NRF2 mutations. The aim of the present review was to highlight findings of how constitutive Nrf2 activation may be a specific biomarker for predicting patients most likely to benefit from classical chemotherapy drugs, overall improving patient survival rate.
\end{abstract}

\section{Contents}

1. Introduction

2. Keap1-Nrf2 pathway

3. Mechanisms of Keap1-Nrf2 pathway deregulation

4. Keap1-Nrf2 pathway disruption and prognosis

5. Co-occurring aberrations

6. Crosstalk between the Keap1-Nrf2 and EGFR pathways

7. Drug resistance and clinical implications

8. Future directions and conclusions

Correspondence to: Dr Raúl Barrera-Rodríguez, Department of Biochemistry and Environmental Medicine, National Institute of Respiratory Diseases, 4502 Calzada de Tlalpan, Col Sec. XVI, Mexico City 14080, Mexico

E-mail: barrerarr@hotmail.com

Key words: lung cancer, non-small cell lung cancer, kelch-like $\mathrm{ECH}$-associated protein 1-nuclear factor erythroid-2-related factor-2 pathway, drug resistance, biomarker

\section{Introduction}

Lung cancer is the leading cause of cancer-related mortality worldwide, with a 5-year overall survival rate of $<15 \%$ (1). Non-small cell lung cancer (NSCLC) is the most common histological type of lung cancer, representing $\sim 85 \%$ of cases, and its incidence has risen steadily over the past few decades, particularly in women (2). Although target-directed therapy has been demonstrated to improve chemotherapy response among NSCLC patients (3), for the numerous NSCLC patients with an unknown mutation status or with no apparent gene mutations, platinum-based regimens are the standard of care recommended by the European Society for Medical Oncology Clinical Practise Guidelines (4); however, chemotherapy for advanced inoperable NSCLC is generally palliative. The major factor contributing to failure of chemotherapy in the treatment of lung cancer is development of drug resistance (5).

The lung is continuously exposed to chemicals and carcinogens that cause oxidative stress to lipids, proteins and DNA. In response, lung cells must activate cytoprotective mechanisms to defend and protect themselves against these insults (6). Lung cancer tumors seem to inherit this ability, which affords them protection against insults that generate oxidative stress or against the toxicity of xenobiotics produced in the microenvironmental conditions of tumour growth (7). The kelch-like ECH-associated protein 1 (Keap1)-nuclear factor erythroid-2-related factor-2 (Nrf2) pathway is a key determinant for cells in coping with oxidative stress (8). In response to environmental or endogenous insults, the Keap1-Nrf2 pathway increases the expression of a series of cytoprotective/defensive proteins that protect cells against oxidative stress and promote cell survival (9).

Previous data indicate that abnormal states of the Keap1-Nrf2 pathway exist in lung cancer (10-12). Elevated Nrf2 levels and Keap1 dysfunction have been frequently identified in lung cancer, and it is possible that they are associated with tumour progression, cytoprotection, resistance to chemotherapeutic drugs and poor prognosis (13).

The current review summarizes recent advancements in our understanding of alterations in the Keap1-Nrf2 pathway in NSCLC, its role in drug resistance, and discusses its usefulness as a potential biomarker. An improved understanding of the role served by the Keap1-Nrf2 pathway in the regulation of 
cytoprotective mechanisms may aid the search for novel anticancer targets that afford decreased tumour defense systems and increased sensitivity to treatments.

\section{Keap1-Nrf2 pathway}

The Keap1-Nrf2 pathway serves a central role in protecting cells from oxidative and/or electrophilic stress. Nrf2, also known as NFE2L2, belonging to the cap'n'collar subfamily of the basic leucine zipper transcription factors, is the primary regulator of the inducible cell defense system, which mediates the expression of $>200$ oxidative stress-related genes (14), including those transcribing antioxidant proteins, proteasome subunits, chaperones, growth factors and their receptors, certain transcription factors, phase I and II detoxification enzymes, and drug efflux pumps that can accelerate the metabolic inactivation of antitumor agents and decrease intracellular drug concentrations $(8,15,16)$.

Nrf2 activity is tightly regulated by Keap1, which is a cytoplasmic adaptor protein of the Cullin3 (Cul3)-based E3-ligase (17). Under normal physiological conditions, Keap1 constitutively targets Nrf2 for polyubiquitination and degradation by the $26 \mathrm{~S}$ proteasome (18). However, upon oxidative stress, Keap1 is inactivated and the ubiquitination of Nrf2 halted, which leads to the accumulation of Nrf2 in the cytoplasm. Consequently, Nrf2 is translocated into the nucleus via the importin- $\alpha 5$ /importin- $\beta 1$ import pathway, where it induces the transcription of a series of antioxidant responsive element (ARE)-responsive genes and ultimately leads to the activation of the defensive system as well as mechanisms of chemoradiation resistance (19) (Fig. 1).

Several KEAP1 and NRF2 gene mutations which disrupt the Keap1-Nrf2 interaction and result in Nrf2 overexpression have been identified in several cancer tissues, including lung, breast, bladder, ovarian and liver, and numerous types of cancer cell line $(10,20-23)$.

\section{Mechanisms of Keap1-Nrf2 pathway deregulation}

Distinct mechanisms have been described for the activation of Nrf2 in NSCLC, which include:

i) Somatic mutations: Gain-of-function mutations in NRF2 and loss-of-function mutations in KEAP1 (8). Initially, KEAP1 gene mutations (G430C and G364C) were identified in a human lung adenocarcinoma cell line (NCI-H1648), each involving a glycine to cysteine substitution in the Kelch-repeat domain of Keap1 (20). These adenocarcinoma cells exhibited reduced affinity of Keap1 to Nrf2 and in consequence a constitutive activation of Nrf2 was observed (18). Subsequent to this, other somatic mutations have also been identified in the Kelch or intervening linker (IVR) domains of the Keap1 protein in NSCLC cell lines (NL20, A549, H460, H1435, H23, H358, H1993, H1395, H838, H1299 and H292) and in tissues from NSCLC patients $(10,11)$.

ii) KEAP1 hypermethylation: DNA methylation by DNA methyltransferases in the promoter region of KEAP1 may affect its expression and hinder its ability to bind to Nrf2, resulting in Nrf2 activation $(12,24)$. Conversely, CpG methylation of the NRF2 promoter appears to downregulate NRF2 expression indirectly $(25,26)$. iii) Accumulation of $\mathrm{p} 21^{\mathrm{Cip} 1 / \mathrm{WAF} 1}$ and $\mathrm{p} 62^{\mathrm{lck}}$ may disrupt the Keap1-Nrf2 complex (27): In response to injuries producing reactive oxygen species (ROS), cells generate an Nrf2-dependent anti-oxidant response in an attempt to repair the ROS- and/or electrophile-induced damages. However, if the ROS provoke DNA damage, the transcription factor $\mathrm{p} 53$ may be activated, which induces cell cycle arrest to allow time for the DNA repair (28). It is this point where the cyclindependent kinase inhibitor $\mathrm{p} 21^{\mathrm{Cip} 1 / \mathrm{WAF} 1}$ (a direct downstream target of p53) may associate with the DLG motif of Nrf2, inhibiting Keap1-dependent Nrf2 ubiquitination, resulting in stabilization of the Nrf2 protein and, ultimately, leading to the enhanced expression of oxidative stress-related genes (29). However, if DNA is unrepaired, a second response of the cell may occur and p53-induced apoptosis is promoted. Under this condition, p53 appears to suppress the transcription of target genes of Nrf2 (30). However, impairment of autophagy in cancer cells is usually accompanied by accumulation of p62/sequestosome 1 protein. This protein, targeted directly onto the Kelch-repeat domain of Keap1 via its STGE motif, may thereby disrupt the Keap1-Nrf2 complex (31). Again, this interaction may cause a decrease in the ubiquitination of $\mathrm{Nrf} 2$ and elicit as a result an increase in Nrf2 stability $(29,32)$.

iv) Transcriptional upregulation of NRF2 by onco-

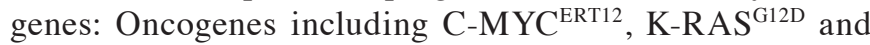
$\mathrm{BRAF}^{\mathrm{V} 619 \mathrm{E}}$ may increase the transcriptional level of NRF2 and NRF2-regulated genes, resulting in a marked increase of cytoprotection of tumour cells (33).

v) Metabolic activation of Nrf2 by Kreb's cycle intermediates: In the Kreb's cycle, fumarate modifies cysteine residues within Keap1, which disrupts the ability to ubiquitinate Nrf2 and in consequence prolongs activation of Nrf2 (34). In addition, defects in fumarate hydratase may stimulate an importin-mediated nuclear transport of Nrf2 and the transcription of antioxidant enzymes through the succination of Keap1 (35). Furthermore, it has been documented that metabolic reprogramming of both catabolic and anabolic pathways appear to be driven by Keap1-Nrf2 aberrations. For example, DeNicola et al (36) demonstrated that activation of Nrf2 regulated serine and glycine metabolism and was linked with clinical aggressiveness in NSCLC, and Mitsuishi et al (37) showed that Nrf2 redirected glucose and glutamine into anabolic pathways, particularly under the sustained activation of phosphatidylinositide 3-kinase (PI3K)-protein kinase B (PKB)/Akt signaling, which was advantageous for proliferation and survival in A549 cells.

vi) Loss of exon 2 in the NRF2 gene: Aberrant NRF2 transcripts may result from exon 2 skipping, which translates an Nrf2 protein isoform missing either the DLG or ETGE motifs of the regulatory Nrf2-ECH homology (Neh)2 domain, resulting in persistent $\mathrm{Nrf} 2$ localization in the nucleus $(38,39)$;

vii) In recent years, there has been recognition that the repression of Nrf2 by Cul1- $\beta$-transducin repeat-containing protein $(\beta-\mathrm{TrCP}) \mathrm{E} 3$ ubiquitin ligase is augmented by glycogen synthase kinase-3 $\beta$ (GSK-3 $\beta$ ). Thus, Nrf 2 may be regulated by GSK-3 $\beta$ through the creation of a DSGIS motif-containing phosphodegron present in the Neh6 domain of Nrf2 that is recognized by Cul13- $\beta$-TrCP (40). Conversely, the phosphorylation of Nrf 2 by GSK-3 $\beta$ may be inhibited by growth factor signaling through the PI3K-Akt pathway (41). 


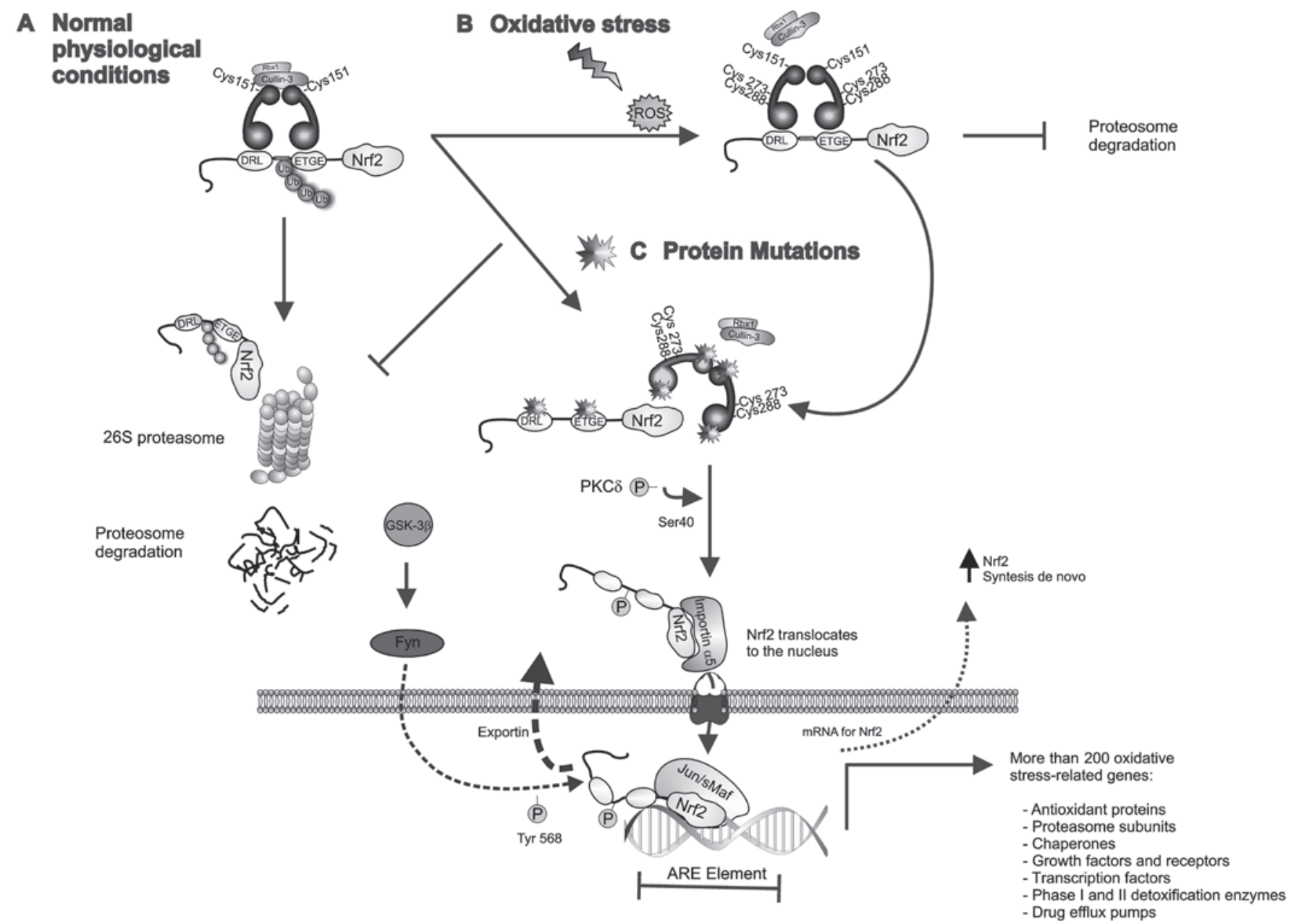

Figure 1. Keap1-Nrf2 pathway. (A) Under physiological/basal conditions, the Keap1-Cullin3 complex promotes ubiquitin E3-ligase activity and proteasomal degradation of Nrf2. (B) Upon exposure to chemicals or ROS, reactive cysteine residues of Keap1 are modified leading to a disruption of the Keap1-Nrf2 complex and the release of Nrf2, which also allows de novo synthesized Nrf2 to accumulate in the cytoplasm. The phosphorylation of Ser- 40 in the Neh2 domain of Nrf2 by PKC $\delta$ results in Nrf2 activation and translocation to the nucleus, where Nrf2-mediated transcriptional activity of ARE-responsive genes is initiated. GSK-3 $\beta$ phosphorylates the tyrosine kinase Fyn and induces its nuclear accumulation. Fyn phosphorylates Nrf2 at tyrosine-568, facilitating its nuclear export and degradation. (C) Loss-of-function mutations in Keap1 or Nrf2 lead to constitutive activation of Nrf2 by disrupting the Keap1-Nrf2 interaction. In the nucleus, Nrf2 heterodimerizes with sMaf proteins and regulates the expression of $>200$ oxidative stress-related genes. ARE, antioxidant response element; Fyn, proto-oncogene Src-family tyrosine kinase; GSK-3ß, glycogen synthase kinase 3; Keap1, kelch-like ECH-associated protein 1; sMaf, musculoaponeurotic fibrosarcoma oncogene; Nrf2, nuclear factor erythroid-2-related factor-2; PKC $\delta$, protein kinase $\mathrm{C}-\delta$; ROS, reactive oxygen species.

\section{Keap1-Nrf2 pathway disruption and prognosis}

There is increasing data to suggest that KEAP1-NRF2 mutational status is associated with poor prognosis and chemotherapeutic resistance in NSCLC (10). For example, Inoue et al (42) examined the expression of Nrf2 by immunohistochemical analyses, in clinical tissue samples from 109 NSCLC cases, and observed that higher nuclear accumulation of Nrf2 correlated with worse lung cancerspecific survival. By immunohistochemistry, Yang et al (43) analyzed the Nrf2 expression status of 60 patients with stage IIIB or IV NSCLC and compared the response to platinum-based treatments in both groups. Although positive staining for Nrf2 was found in nearly all cases to varying degrees, patients with stage IV disease exhibited higher Nrf2 expression than patients with stage IIIB disease $(\mathrm{P}=0.017)$. Interestingly, patients with $<75 \%$ positive staining achieved a higher response rate than those with $75-100 \%$ positive staining $(\mathrm{P}=0.003 ; \mathrm{r}=0.447)$, suggesting that $\mathrm{Nrf} 2$ expression may be a useful index to predict the efficacy of platinumbased treatments (43).

Interestingly, the results of several studies of NSCLC tumour samples have also indicated that the occurrence of NRF2 or KEAP1 mutation is mutually exclusive and associated with different histologies. Solis et al (44) studied 304 tumour tissue samples [190 adenocarcinomas (ADCs) and 114 squamous cell carcinomas (SqCCs)] following adjuvant treatment. They detected that nuclear Nrf2 expression in $26 \%$ of the NSCLC samples was significantly more common in SqCC (38\%) than in ADC (18\%; $\mathrm{P}<0.0001)$ and established an association of a nuclear Nrf2 abundance with worse progression-free survival. Li et al (45) reported that the frequency of Keap1 alterations was significantly higher in papillary ADC tumors (60\%) than that reported previously for NSCLC (3-19\%) (46). In a recent study, Frank et al (47) analyzed the tumour tissues of 1,391 patients with NSCLC and identified that the frequency for Nrf2 mutations was 3.5\% ( $\mathrm{n}=49)$, while for Keap1 mutations was $11.3 \%(\mathrm{n}=157)$. Nrf2 mutations were 


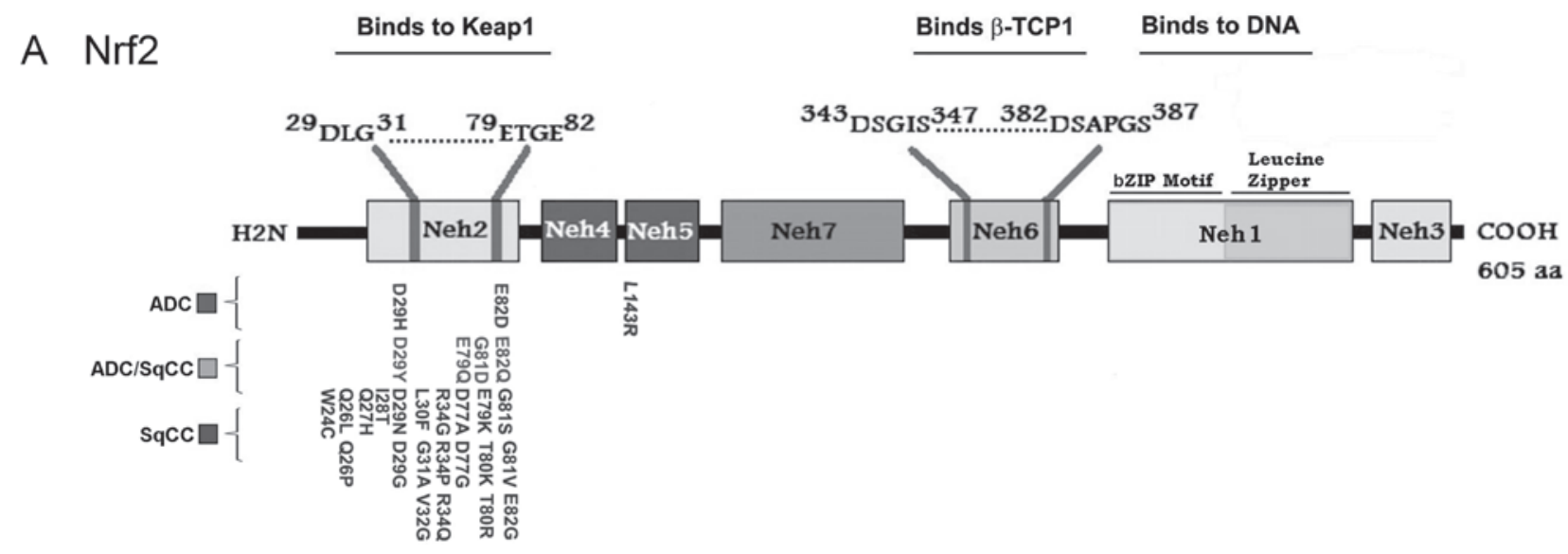

B Keap1 Binds to Cullin-3 Binds to Nrf2

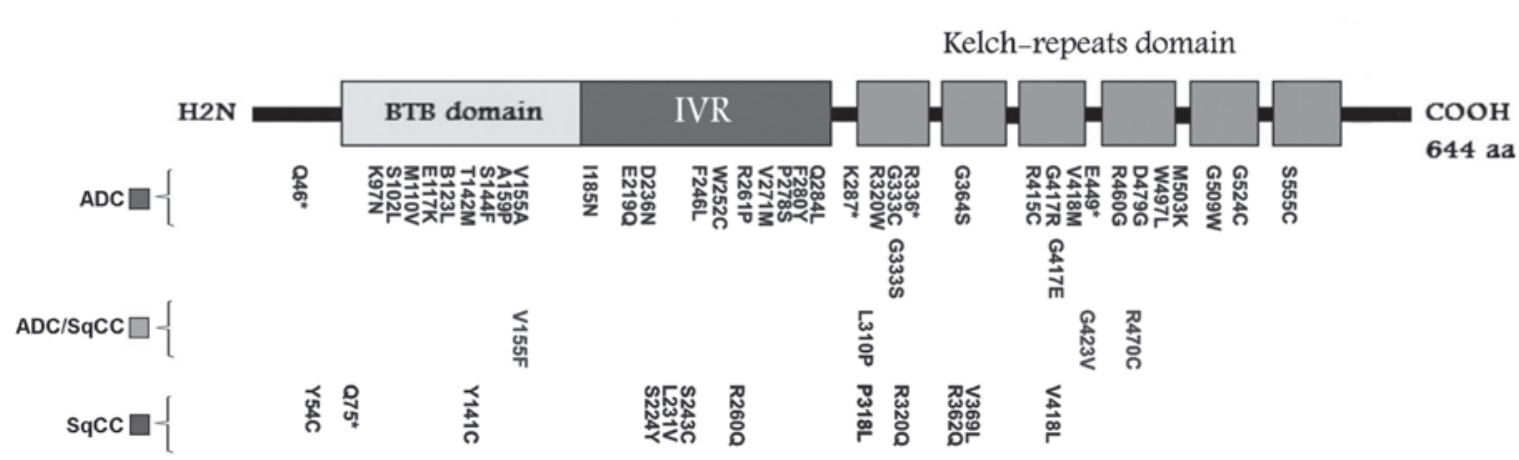

Figure 2. Schematic representation of domain architecture of the Keap1 and Nrf2 proteins and mutations found in NSCLC. (A) Human NRF2 is a polypeptide of 605 amino acids and contains 7 Neh domains. Neh1 contains the signature CNC motif, which is a highly conserved bZIP domain for DNA binding. The Neh1 CNC-bZIP domain is responsible for dimerization with sMaf protein and is required for binding to ARE sequences in DNA; the Neh2 domain controls the interaction with Keap1 through the DLG and ETGE motifs; the Neh6 domain contains two binding sites (DSGIS and DSAPGS motifs), and the phosphorylation of the DSGIS motif by GSK-3 $\beta$ increases binding ability to the $\beta$-TrCP1 adaptor protein. (B) Human Keap1 is a polypeptide of 644 amino acids. The BTB domain is required for the formation of Keap1 homodimers as well as the recruitment of Cul3-based E3-ligase. The Kelch-repeat domain controls Nrf2 interaction. The region between the BTB and Kelch repeat domains constitutes the IVR. Amino acid positions of the identified mutations of Nrf2 and Keap1 are shown for $\mathrm{ADC}, \mathrm{ADC} / \mathrm{SqCC}$ and $\mathrm{SqCC}$. All the indicated mutations are listed in the Catalogue of Somatic Mutations in Cancer database (http://cancer. sanger.ac.uk/cosmic). ADC, adenocarcinoma; ADC/SqCC, adenocarcinoma/squamous cell carcinoma; ARE, antioxidant response element; bZIP, basic leucine zipper; BTB, broad complex, tram-track and bric-a-brac; CNC, cap'n'collar; Cul3, Cullin3; GSK-3 $\beta$, glycogen synthase kinase 3; IVR, intervening linker; KEAP1, kelch-like ECH-associated protein 1; sMaf, musculoaponeurotic fibrosarcoma oncogene; Neh, Nrf2-ECH homologous structure; NRF2, nuclear factor erythroid-2-related factor-2; SqCC, squamous cell carcinoma; $\beta$-TrCP1, $\beta$-T-complex protein 1.

most frequent in SqCC (59.2\%) whereas Keap1 mutations were predominantly detected in ADC (72.2\%). Furthermore, patients with tumors containing KEAP1 mutation presented a worse Eastern Cooperative Oncology Group performance status and the response on application to different chemotherapy regimens was notably poor (47). A large-scale genomic study involving The Cancer Genome Atlas Research Network examined the exome sequences and copy number profiles of $660 \mathrm{ADC}$ and $484 \mathrm{SqCC}$ tumour/normal tissue pairs (48). This report estimated a KEAP1-NRF2 mutation frequency of 34\% in 178 tumors from affected individuals and revealed that the KEAP1 gene was significantly mutated exclusively in ADC, whereas NRF2 was significantly mutated in SqCC. CUL3, the protein product of which is a known interaction partner of Keap1, also reached statistical significance as a mutated gene in the lung SqCC cohort (48).

Studies have also investigated whether the frequency of KEAP1-NRF2 mutations differs by race and ethnicity. Ohta et al (11) reported loss of heterozygosity ( $\mathrm{LOH})$ in
KEAP1 in 5 of $65(8 \%)$ Japanese patients with lung cancer, and Singh et al (10) performed a systematic analysis of the KEAP1 genomic locus in a Caucasian population of 54 cases of NSCLC samples, and in 12 lung cancer cell lines, in which deletions, insertions, missense mutations of KEAP1 and LOH at 19p13.2 were commonly found. The KEAP1 somatic mutations were detected in 19\% (10 of 54) of all lung cancer cases and in $26 \%$ (9 of 35) of ADC cases (10).

Genomic profiling analysis has suggested the prevalence of NRF2 exon deletions to be $1-2 \%$ in a panel of 113 NSCLC cell lines (38), and the existence of two NRF2 mutation 'hot-spots' in $\sim 10 \%$ of patients with SqCC, which may enable the transcription factor to evade Keap1-mediated repression $(38,49)$. Besides, the mutation burden in Keap1 is spread across the protein domains, while those in Nrf2 only occur in codons for amino acids around the DLG and ETGE motifs (the domains of Nrf2 that interact with Keap1) $(10,11,39,50)$ (Fig. 2).

Despite these data, the frequency of KEAP1 or NRF2 mutation in NSCLC remains uncertain, which thus warrants further 
studies to provide discriminating differences between the NSCLC subtypes as well as a comparison between racial/ethnic groups.

\section{Co-occurring aberrations}

Comparative genomic analyses have determined that co-occurring genomic aberrations are present in $83.7 \%$ of patients with tumors containing a mutation in Nrf2 and $87.3 \%$ of those with a mutation in Keap1 (47). Mutations in the gene for p53 (TP53) are the most common concurrent aberration, with a frequency of $40.8 \%$ in tumors harboring mutations in Nrf2 and $44.9 \%$ in tumors with mutations in Keapl. Similar distribution patterns have been observed for epidermal growth factor receptor (EGFR) mutations, with a frequency of $6.1 \%$ reported in tumors with mutation in Nrf2 and 6.3\% in those with mutation in Keap1. For c-MET amplification, a mutation frequency of $26.3 \%$ has been observed in tumors with Nrf2 mutation, while for Keap1 mutation the frequency was $18.3 \%$ (47). NSCLC with Keap1 mutation which also harbors an activating KRAS mutation is associated with worse prognosis compared with KRAS-mutated patients without Keap1 mutation (51). Interestingly, patients with ADC that have high mutational load and which could benefit from anti-programmed cell death-1 treatment have shown a high prevalence of Keap1 mutations (52). By contrast, SqCC and mutations in Nrf2 have been associated with high programmed death-ligand 1 expression (53).

Altogether these studies further suggest that in NSCLC, the mutations in KEAP1 or NRF2 may each give rise to a different subset of cancer, opening the opportunity to select a novel series of druggable molecules distinct between the two main lung cancer histological types, for overall improved treatment. Therefore, it appears necessary to implement next-generation sequencing (NGS)-based multiplex diagnostics for cancer, which is capable of capturing and amplifying $~ 10,000$ human exons in a single multiplex reaction. Through use of such novel technology, an improved understanding may be gained of the molecular interactions of mutations in KEAP1 and NRF2 with co-occurring, targetable genetic aberrations.

\section{Crosstalk between the Keap1-Nrf2 and EGFR pathways}

In lung ADC the tyrosine kinase activity of EGFR is frequently overexpressed or highly activated and has been associated with growth, survival and therapeutic resistance (54). EGFR aberrations can overactivate downstream pro-oncogenic signaling pathways, including the PI3K/Akt and mitogenactivated protein kinase/extracellular signal-regulated kinase pathways, leading to cell growth and proliferation (55). As a result, EGFR has become viewed as an important specific molecule for targeted therapy with specific tyrosine kinase inhibitors (TKIs) (56). In cancer cells, EGFR-mediated stimulation of the PI3K/Akt pathway may inhibit the constitutive activity of GSK- $3 \beta$, decreasing the GSK-3 $\beta$-dependent degradation of Nrf2, enabling it to translocate to the nucleus, where Nrf2-mediated transcriptional activity of cytoprotective genes is initiated (57). Conversely, the activation of GSK-3 $\beta$ by inhibition of PI3K/Akt was reported to decrease Nrf2 protein levels in human A549 lung cells that lack functional Keap1 (48). Activation of the PI3/Akt pathway provides a ratio- nalized explanation of how ARE-driven genes may be induced by aberrant activity of growth factor receptors, and of why this pathway may be implicated as an important mechanism in resistance to EGFR inhibitors (58).

Furthermore, EGFR itself may directly regulate Nrf2 activity in cancer cells. Huo et al (59) showed that nuclear EGFR induced phosphorylation and ubiquitination of Keap1, leading to stabilization of Nrf2 and stimulation of transcriptional activity, which contributed to cancer cell resistance to chemotherapy. Thus, the function of the EGFR-PI3K-AKT pathway in regulating the Keap1-Nrf2 axis may have an important role not only in cancer cell growth but also in the expression of genes that confer drug resistance in human cancers (60).

Notably, in lung tumors, mutations of EGFR or overactivity of Nrf2 appear to be mutually exclusive (61); it has been observed that the frequency of nuclear Nrf2 expression is significantly higher in EGFR wild-type ADC (21\%) than in tumors with EGFR mutation $(0 \%)(44,62)$. In this regard, it has been speculated that the lack of nuclear Nrf2 expression in ADC containing EGFR mutations may be an important factor that contributes to the chemosensitivity observed with platinum-based chemotherapy (44). This crosstalk between the EGFR-PI3K-AKT and Keap1-Nrf2 pathways may explain why for certain cases of NSCLC, mortality rate remains among the highest of all cancers, despite the availability of improved therapeutics including EGFR-TKIs (56).

The interaction of mutations in KEAP1 and NRF2 with co-occurring targetable genetic aberrations requires an improved understanding, and highlights the need for NGS-based molecular cancer diagnostics to cover co-occurring mutations at least in the setting of clinical research.

\section{Drug resistance and clinical implications}

There is data to suggest that the activation of the Keap1-Nrf2 pathway is associated with the emergence of resistance to chemotherapy drugs via transcriptional activation of genes conferring resistance to such drugs, including: Multidrug resistance-associated protein 1 [also known as ATP binding cassette (ABC) subfamily $C$ member 1$]$ and $\gamma$-glutamylcysteine synthetase genes, which may be involved in resistance against cisplatin and alkylating agents $(63,64)$; breast cancer resistance protein (also known as ABC subfamily G member 2), which is considered to mediate the efflux of gefitinib (an EGFR inhibitor), conferring resistance to TKI regardless of EGFR mutation (65-67); and several other cytoprotective genes (heme oxygenase 1, NAD $(\mathrm{P}) \mathrm{H}$ quinone dehydrogenase 1 and glutathione S-transferases) (68).

In support of these observations, it has been reported that the inhibition of Nrf2 in lung cancer cells resulted in enhanced intracellular accumulation of carboplatin and etoposide, and consequently in enhanced chemotherapy-induced cell death (69,70). Furthermore, Tian et al (71) reported that small interfering RNA knockdown of Nrf2 significantly disrupted Nrf2 signaling in vitro and led to sensitization of the H292 cell line to platinum-based drugs, and Singh et al (70) demonstrated that xenografts derived from Nrf2-silenced lung cancer cells had an enhanced response to carboplatin in vivo compared with control knockdown cells. 
In clinical practice, the repercussion of deregulation in the Keap1-Nrf2 pathway is a negative effect for patients with NSCLC, generally manifesting as reduction in overall survival and poorer prognosis. Several reports support this; for example, Solis et al (44) reported that Nrf2 overexpression $[\mathrm{P}=0.0139$; hazard ratio (HR), 1.75] or low or absent Keap1 expression ( $\mathrm{P}=0.0181 ; \mathrm{HR}, 2.09)$ was associated with worse overall survival in SqCC. Takahashi et al (62) identified that KEAP1 gene mutations were likely associated with a worse prognosis and lower postoperative disease-free survival rate in pathological stage I-II NSCLC. These observations were also confirmed in other reports, where the nuclear expression of Nrf2 was indicated to serve a role in resistance to platinum-based treatment in $\mathrm{SqCC}(61,72,73)$. A previous meta-analysis of microarray data on the expression signatures of 240 NRF2-mediated genes identified a group of 50 genes that predicted a worse clinical outcome in $60 \%$ of NSCLC cohorts analyzed (72). In a recent study of patients with NSCLC, the median overall survival of patients with Keap1 mutation was 19.1 months (95\% CI, 1.8-36.3 months), and 14.0 months (95\% CI, 5.6-22.3 months) for those patients with Nrf2 mutation (47).

Overall, the available data indicates that Keap1-Nrf2 pathway activation serves an important role in the acquisition of resistance to chemotherapy in NSCLC, and provides an explanation for the poor outcomes observed clinically. It has therefore been suggested that the dysregulation of Keap1-Nrf2 pathway may be a clinically useful biomarker of prognosis in lung cancer patients $(74,75)$.

\section{Future directions and conclusions}

In recent years, pharmaceutical companies have focused on Keap1-Nrf2 pathway targets in order to identify novel and effective molecules capable of inhibiting the Keap1-Nrf2 interaction (76). An obvious advantage of targeting Keap1 or Nrf2 molecules would be the expected increase in effectiveness of standard antitumor chemotherapies; targeting of the detoxification pathways involved in detoxification of various chemotherapy drugs would also be required to reduce the possibility of alternative or redundant detoxification pathway activation. Two reports in the last 5 years have provided a comprehensive summary of current literature relevant to small-molecule modulators of the Keap1-Nrf2 pathway and their usefulness as potential preventive and therapeutic agents (77,78).

Recently, the inhibition of NRF2 has become a promising therapeutic approach for cancers displaying Nrf2 overactivation, and consequently, the clinical implementation of Nrf2 inhibitors in NSCLC patients with Keap1-Nrf2 pathway deregulation may be a useful therapeutic strategy (76). There have been few Nrf2 inhibitors identified to date. Brusatol, a quassinoid isolated from the Brucea javanica shrub, has been identified as a unique inhibitor of the Nrf2 through enhancing ubiquitination and degradation of Nrf2 (79). Notably, treatment with brusatol sensitized a broad spectrum of cancer cells to antitumor drugs, reduced tumour burden and improved survival in murine A549 xenograft models $(80,81)$. Nevertheless, while the use of targeted inhibitors may be a novel therapeutic strategy to treat several types of cancer, it is noteworthy that administration of systemic NRF2 inhibitors may have undesirable effects on cancer patients, considering the central roles of NRF2 in cytoprotection (82).

Novel potential therapeutic targets in cancers exhibiting Keap1-Nrf2 pathway deregulation are being identified in addition to Nrf2 inhibitors. Some of these targets, including the glutathione synthesis, serine synthesis and pentose phosphate pathways, and IL-11, are direct or indirect downstream effectors of Nrf2 in mediating malignant phenotypes. However, efforts are still required to search for novel compounds and engineered small molecules that target Nrf2 more specifically, and to establish Nrf2 inhibitors suitable for safe clinical use and temporary use as a sensitizer in chemo- and radiotherapy regimens.

To date, considerable progress has been made to understand the mechanisms involved in the fine regulation of the Keap1-Nrf2 pathway and its downstream genes. However, due to the complexity of the cross-talk between Nrf2 and its numerous signaling-network partners, further studies are still required to determine the optimum markers for predicting the clinical outcome of patients with NSCLC. An improved understanding of the molecular mechanisms activating the Keap1-Nrf2 pathway may provide a basis for improvement in the selection of patients with poor prognosis, to be treated only with supportive care and thus avoiding unnecessary adverse effects and complications of systemic chemotherapy.

In conclusion, the findings reviewed herein suggest that evaluation of Keap1-Nrf2 pathway dysregulation in patients with NSCLC may provide predictive biomarkers of chemotherapy resistance and improve the accuracy of diagnosis.

\section{Acknowledgements}

The author would like to thank Dr Francisco Hernandez Gómez-Crespo at the Department of Biochemistry and Environmental Medicine, National Institute of Respiratory Diseases (Mexico City, Mexico), for his critique of this manuscript. Thanks are also afforded to Mrs Cristina Wilson at 'Gastro One' medical clinic (Memphis, TN, USA), for her editing of the English language.

\section{Funding}

Not applicable.

\section{Availability of data and materials}

Not applicable.

\section{Authors' contributions}

RBR was responsible for all aspects involved in the design, performance and writing of the literature review.

\section{Ethics approval and consent to participate}

Not applicable.

\section{Consent for publication}

Not applicable. 


\section{Competing interests}

The author declares that they have no competing interests.

\section{References}

1. Siegel RL, Miller KD and Jemal A: Cancer Statistics, 2017. CA Cancer J Clin 67: 7-30, 2017.

2. Cheng TY, Cramb SM, Baade PD, Youlden DR, Nwogu C and Reid ME: The international epidemiology of lung cancer: Latest trends, disparities, and tumor characteristics. J Thorac Oncol 11: $1653-1671,2016$

3. Forde PM and Ettinger DS: Targeted therapy for non-small-cell lung cancer: Past, present and future. Expert Rev Anticancer Ther 13: 745-758, 2013

4. Novello S, Barlesi F, Califano R, Cufer T, Ekman S, Levra MG, Kerr K, Popat S, Reck M, Senan S, et al; ESMO Guidelines Committee: Metastatic non-small-cell lung cancer: ESMO Clinical Practice Guidelines for diagnosis, treatment and follow-up. Ann Oncol 27 (Suppl 5): v1-v27, 2016.

5. Kim ES: Chemotherapy Resistance in Lung Cancer. Adv Exp Med Biol 893: 189-209, 2016.

6. Rahman I, Biswas SK and Kode A: Oxidant and antioxidant balance in the airways and airway diseases. Eur J Pharmacol 533 222-239, 2006

7. Zhang JY, Wang Y and Prakash C: Xenobiotic-metabolizing enzymes in human lung. Curr Drug Metab 7: 939-948, 2006.

8. Jaramillo MC and Zhang DD: The emerging role of the Nrf2Keap1 signaling pathway in cancer. Genes Dev 27: 2179-2191, 2013

9. Ma Q: Role of nrf2 in oxidative stress and toxicity. Annu Rev Pharmacol Toxicol 53: 401-426, 2013.

10. Singh A, Misra V, Thimmulappa RK, Lee H, Ames S, Hoque MO, Herman JG, Baylin SB, Sidransky D, Gabrielson E, et al: Dysfunctional KEAP1-NRF2 interaction in non-small-cell lung cancer. PLoS Med 3: e420, 2006.

11. Ohta T, Iijima K, Miyamoto M, Nakahara I, Tanaka H, Ohtsuji M, Suzuki T, Kobayashi A, Yokota J, Sakiyama T, et al: Loss of Keap1 function activates Nrf2 and provides advantages for lung cancer cell growth. Cancer Res 68: 1303-1309, 2008.

12. Wang R, An J, Ji F, Jiao H, Sun H and Zhou D: Hypermethylation of the Keapl gene in human lung cancer cell lines and lung cancer tissues. Biochem Biophys Res Commun 373: 151-154, 2008.

13. Vollrath V, Wielandt AM, Iruretagoyena $M$ and Chianale $J$ Role of Nrf2 in the regulation of the Mrp2 (ABCC2) gene. Biochem J 395: 599-609, 2006.

14. Lu MC, Ji JA, Jiang ZY and You QD: The Keap1-Nrf2-ARE pathway as a potential preventive and therapeutic target: An update. Med Res Rev 36: 924-963, 2016.

15. Sporn MB and Liby KT: NRF2 and cancer: The good, the bad and the importance of context. Nat Rev Cancer 12: 564-571, 2012

16. Villeneuve NF, Lau A and Zhang DD: Regulation of the Nrf2-Keap1 antioxidant response by the ubiquitin proteasome system: An insight into cullin-ring ubiquitin ligases. Antioxid Redox Signal 13: 1699-1712, 2010.

17. Menegon S, Columbano A and Giordano S: The dual roles of NRF2 in cancer. Trends Mol Med 22: 578-593, 2016.

18. Kobayashi A, Kang MI, Okawa H, Ohtsuji M, Zenke Y, Chiba T, Igarashi $\mathrm{K}$ and Yamamoto $\mathrm{M}$ : Oxidative stress sensor Keap functions as an adaptor for Cul3-based E3 ligase to regulate proteasomal degradation of Nrf2. Mol Cell Biol 24: 7130-7139, 2004

19. Theodore M, Kawai Y, Yang J, Kleshchenko Y, Reddy SP, Villalta F and Arinze IJ: Multiple nuclear localization signals function in the nuclear import of the transcription factor Nrf2. J Biol Chem 283: 8984-8994, 2008.

20. Padmanabhan B, Tong KI, Ohta T, Nakamura Y, Scharlock M, Ohtsuji M,Kang MI, Kobayashi A, Yokoyama S and Yamamoto M: Structural basis for defects of Keap1 activity provoked by its point mutations in lung cancer. Mol Cell 21: 689-700, 2006.

21. Kim YR, Oh JE, Kim MS, Kang MR, Park SW, Han JY, Eom HS Yoo NJ and Lee SH: Oncogenic NRF2 mutations in squamous cell carcinomas of oesophagus and skin. J Pathol 220: 446-451, 2010.

22. Nioi P and Nguyen T: A mutation of Keap1 found in breast cancer impairs its ability to repress Nrf2 activity. Biochem Biophys Res Commun 362: 816-821, 2007

23. Taguchi $\mathrm{K}$ and Yamamoto M: The KEAP1-NRF2 system in cancer. Front Oncol 7: 85, 2017.
24. Muscarella LA,Parrella P,D'Alessandro V, la Torre A, Barbano R, Fontana A, Tancredi A, Guarnieri V, Balsamo T, Coco M, et al: Frequent epigenetics inactivation of KEAP1 gene in non-small cell lung cancer. Epigenetics 6: 710-719, 2011.

25. Khor TO, Fuentes F, Shu L, Paredes-Gonzalez X, Yang AY, Liu Y, Smiraglia DJ, Yegnasubramanian S, Nelson WG and Kong AN: Epigenetic DNA methylation of antioxidative stress regulator NRF2 in human prostate cancer. Cancer Prev Res (Phila) 7: 1186-1197, 2014

26. Guo Y, Yu S, Zhang $C$ and Kong AN: Epigenetic regulation of Keap1-Nrf2 signaling. Free Radic Biol Med 88: 337-349, 2015.

27. Chen W, Sun Z, Wang XJ, Jiang T, Huang Z, Fang D and Zhang DD: Direct interaction between Nrf2 and p21(Cip1/WAF1) upregulates the Nrf2-mediated antioxidant response. Mol Cell 34: 663-673, 2009.

28. Williams AB and Schumacher B: p53 in the DNA-damage-repair process. Cold Spring Harb Perspect Med 6: a026070, 2016.

29. Komatsu M, Kurokawa H, Waguri S, Taguchi K, Kobayashi A, Ichimura Y, Sou YS, Ueno I, Sakamoto A, Tong KI, et al: The selective autophagy substrate p62 activates the stress responsive transcription factor Nrf2 through inactivation of Keap1. Nat Cell Biol 12: 213-223, 2010.

30. Faraonio R, Vergara P,Di Marzo D,Pierantoni MG,Napolitano M, Russo T and Cimino F: p53 suppresses the Nrf2-dependent transcription of antioxidant response genes. J Biol Chem 281: 39776-39784, 2006

31. Ichimura Y, Waguri S, Sou YS, Kageyama S, Hasegawa J, Ishimura R, Saito T, Yang Y, Kouno T, Fukutomi T, et al: Phosphorylation of p62 activates the Keap1-Nrf2 pathway during selective autophagy. Mol Cell 51: 618-631, 2013.

32. Taguchi K, Fujikawa N, Komatsu M, Ishii T, Unno M, Akaike T, Motohashi $\mathrm{H}$ and Yamamoto M: Keapl degradation by autophagy for the maintenance of redox homeostasis. Proc Natl Acad Sci USA 109: 13561-13566, 2012.

33. DeNicola GM, Karreth FA, Humpton TJ, Gopinathan A, Wei C, Frese K, Mangal D, Yu KH, Yeo CJ, Calhoun ES, et al: Oncogeneinduced Nrf2 transcription promotes ROS detoxification and tumorigenesis. Nature 475: 106-109, 2011.

34. Kinch L, Grishin NV and Brugarolas J: Succination of Keap1 and activation of Nrf2-dependent antioxidant pathways in FH-deficient papillary renal cell carcinoma type 2. Cancer Cell 20: 418-420, 2011.

35. Adam J, Hatipoglu E, O'Flaherty L, Ternette N, Sahgal N, Lockstone H, Baban D, Nye E, Stamp GW, Wolhuter K, et al: Renal cyst formation in Fh1-deficient mice is independent of the Hif/Phd pathway: Roles for fumarate in KEAP1 succination and Nrf2 signaling. Cancer Cell 20: 524-537, 2011.

36. DeNicola GM, Chen PH, Mullarky E, Sudderth JA, Hu Z, Wu D, Tang H, Xie Y, Asara JM, Huffman KE, et al: NRF2 regulates serine biosynthesis in non-small cell lung cancer. Nat Genet 47: $1475-1481,2015$

37. Mitsuishi Y, Taguchi K, Kawatani Y, Shibata T, Nukiwa T, Aburatani $\mathrm{H}$, Yamamoto $\mathrm{M}$ and Motohashi $\mathrm{H}$ : Nrf2 redirects glucose and glutamine into anabolic pathways in metabolic reprogramming. Cancer Cell 22: 66-79, 2012.

38. Goldstein LD, Lee J, Gnad F, Klijn C, Schaub A, Reeder J, Daemen A, Bakalarski CE, Holcomb T, Shames DS, et al: Recurrent loss of NFE2L2 Exon 2 is a mechanism for Nrf2 pathway activation in human cancers. Cell Rep 16: 2605-2617, 2016.

39. Shibata T, Ohta T, Tong KI, Kokubu A, Odogawa R, Tsuta K, Asamura H, Yamamoto $M$ and Hirohashi S: Cancer related mutations in NRF2 impair its recognition by Keap1-Cul3 E3 ligase and promote malignancy. Proc Natl Acad Sci USA 105: 13568-13573, 2008.

40. Chowdhry S, Zhang Y, McMahon M, Sutherland C, Cuadrado A and Hayes JD: Nrf2 is controlled by two distinct $\beta$-TrCP recognition motifs in its Neh6 domain, one of which can be modulated by GSK-3 activity. Oncogene 32: 3765-3781, 2013.

41. KoundourosNand PoulogiannisG:Phosphoinositide3-kinase/Akt signaling and redox metabolism in cancer. Front Oncol 8: 160, 2018.

42. Inoue D, Suzuki T, Mitsuishi Y, Miki Y, Suzuki S, Sugawara S, Watanabe M, Sakurada A, Endo C, Uruno A, et al: Accumulation of p62/SQSTM1 is associated with poor prognosis in patients with lung adenocarcinoma. Cancer Sci 103: 760-766, 2012.

43. Yang H, Wang W, Zhang Y, Zhao J, Lin E, Gao J and He J: The role of NF-E2-related factor 2 in predicting chemoresistance and prognosis in advanced non-small-cell lung cancer. Clin Lung Cancer 12: 166-171, 2011. 
44. Solis LM, Behrens C, Dong W, Suraokar M, Ozburn NC, Moran CA, Corvalan AH, Biswal S, Swisher SG, Bekele BN, et al: Nrf2 and Keap1 abnormalities in non-small cell lung carcinoma and association with clinicopathologic features. Clin Cancer Res 16: 3743-3753, 2010.

45. Li QK, Singh A, Biswal S, Askin F and Gabrielson E: KEAP1 gene mutations and NRF2 activation are common in pulmonary papillary adenocarcinoma. J Hum Genet 56: 230-234, 2011.

46. Kim Y, Hammerman PS, Kim J, Yoon JA, Lee Y, Sun JM, Wilkerson MD, Pedamallu CS, Cibulskis K, Yoo YK, et al: Integrative and comparative genomic analysis of lung squamous cell carcinomas in East Asian patients. J Clin Oncol 32: 121-128, 2014.

47. Frank R, Scheffler M, Merkelbach-Bruse S, Ihle MA, Kron A Rauer M, Ueckeroth F, König K, Michels S, Fischer R, et al Clinical and pathological characteristics of KEAP1- and NFE2L2-mutated non-small cell lung carcinoma (NSCLC). Clin Cancer Res 24: 3087-3096, 2018.

48. Campbell JD, Alexandrov A, Kim J, Wala J, Berger AH, Pedamallu CS, Shukla SA, Guo G, Brooks AN, Murray BA, et al; Cancer Genome Atlas Research Network: Distinct patterns of somatic genome alterations in lung adenocarcinomas and squamous cell carcinomas. Nat Genet 48: 607-616, 2016.

49. Hayes JD and McMahon M: NRF2 and KEAP1 mutations: Permanent activation of an adaptive response in cancer. Trends Biochem Sci 34: 176-188, 2009.

50. Cancer Genome Atlas Research Network: Comprehensive genomic characterization of squamous cell lung cancers. Nature 489: 519-525, 2012

51. Riely GJ, Jordan E, Kim HR, Yu HA, Berger MF and Solit DB: Association of outcomes and co-occurring genomic alterations in patients with KRAS-mutant non-small cell lung cancer. J Clin Oncol 34: 9019, 2016.

52. Rizvi NA, Hellmann MD, Snyder A, Kvistborg P, Makarov V, Havel JJ, Lee W, Yuan J, Wong P, Ho TS, et al: Cancer immunology. Mutational landscape determines sensitivity to PD-1 blockade in non-small cell lung cancer. Science 348: 124-128, 2015.

53. Scheel AH, Ansén S, Schultheis AM, Scheffler M, Fischer RN, Michels S, Hellmich M, George J, Zander T, Brockmann M, et al: PD-L1 expression in non-small cell lung cancer: Correlations with genetic alterations. Oncoimmunology 5: e1131379, 2016.

54. Bethune G, Bethune D, Ridgway $\mathrm{N}$ and Xu Z: Epidermal growth factor receptor (EGFR) in lung cancer: An overview and update. $\mathrm{J}$ Thorac Dis 2: 48-51, 2010.

55. Seshacharyulu P, Ponnusamy MP, Haridas D, Jain M, Ganti AK and Batra SK: Targeting the EGFR signaling pathway in cancer therapy. Expert Opin Ther Targets 16: 15-31, 2012.

56. Kobayashi S, Boggon TJ, Dayaram T, Jänne PA, Kocher O, Meyerson M, Johnson BE, Eck MJ, Tenen DG and Halmos B: EGFR mutation and resistance of non-small-cell lung cancer to gefitinib. N Engl J Med 352: 786-792, 2005.

57. Papaiahgari S, Yerrapureddy A, Hassoun PM, Garcia JG, Birukov KG and Reddy SP: EGFR-activated signaling and actin remodeling regulate cyclic stretch-induced NRF2-ARE activation. Am J Respir Cell Mol Biol 36: 304-312, 2007.

58. Jacobsen K, Bertran-Alamillo J, Molina MA, Teixidó C, Karachaliou N, Pedersen MH, Castellví J, Garzón M, CodonyServat C, Codony-Servat J, et al: Convergent Akt activation drives acquired EGFR inhibitor resistance in lung cancer. Nat Commun 8: 410, 2017.

59. Huo L, Li CW, Huang TH, Lam YC, Xia W, Tu C, Chang WC, Hsu JL, Lee DF, Nie L, et al: Activation of Keap1/Nrf2 signaling pathway by nuclear epidermal growth factor receptor in cancer cells. Am J Transl Res 6: 649-663, 2014.

60. Denduluri SK, Idowu O, Wang Z, Liao Z, Yan Z, Mohammed MK, Ye J, Wei Q, Wang J, Zhao L, et al: Insulin-like growth factor (IGF) signaling in tumorigenesis and the development of cancer drug resistance. Genes Dis 2: 13-25, 2015

61. Sasaki H, Hikosaka Y, Okuda K, Kawano O, Moriyama S, Yano M and Fujii Y: NFE2L2 gene mutation in male Japanese squamous cell carcinoma of the lung. J Thorac Oncol 5: 786-789, 2010

62. Takahashi T, Sonobe M, Menju T, Nakayama E, Mino N, Iwakiri S Nagai S, Sato K, Miyahara R, Okubo K, et al: Mutations in Keap are a potential prognostic factor in resected non-small cell lung cancer. J Surg Oncol 101: 500-506, 2010.

63. Ishikawa T, Bao JJ, Yamane Y, Akimaru K, Frindrich K, Wright CD and Kuo MT: Coordinated induction of MRP/GS-X pump and gamma-glutamylcysteine synthetase by heavy metals in human leukemia cells. J Biol Chem 271: 14981-14988, 1996.
64. Adachi T, Nakagawa H, Chung I, Hagiya Y, Hoshijima K, Noguchi N, Kuo MT and Ishikawa T: Nrf2-dependent and -independent induction of $\mathrm{ABC}$ transporters $\mathrm{ABCC} 1, \mathrm{ABCC} 2$, and ABCG2 in HepG2 cells under oxidative stress. J Exp Ther Oncol 6: 335-348, 2007

65. Singh A, Wu H,Zhang P, Happel C, Ma J and Biswal S: Expression of ABCG2 (BCRP) is regulated by Nrf2 in cancer cells that confers side population and chemoresistance phenotype. Mol Cancer Ther 9: 2365-2376, 2010.

66. Saito H, Hirano H, Nakagawa H, Fukami T, Oosumi K, Murakami K, Kimura H, Kouchi T, Konomi M, Tao E, et al: A new strategy of high-speed screening and quantitative structureactivity relationship analysis to evaluate human ATP-binding cassette transporter ABCG2-drug interactions. J Pharmacol Exp Ther 317: 1114-1124, 2006.

67. Meyer zu Schwabedissen HE, Grube M, Dreisbach A, Jedlitschky G, Meissner K, Linnemann K, Fusch C, Ritter CA, Völker U and Kroemer HK: Epidermal growth factor-mediated activation of the map kinase cascade results in altered expression and function of ABCG2 (BCRP). Drug Metab Dispos 34: 524-533, 2006.

68. Hayes JD, McMahon M, Chowdhry S and Dinkova-Kostova AT: Cancer chemoprevention mechanisms mediated through the Keap1-Nrf2 pathway. Antioxid Redox Signal 13: 1713-1748, 2010.

69. Zhang P, Singh A, Yegnasubramanian S, Esopi D, Kombairaju P, Bodas M, Wu H, Bova SG and Biswal S: Loss of Kelch-like $\mathrm{ECH}$-associated protein 1 function in prostate cancer cells causes chemoresistance and radioresistance and promotes tumor growth. Mol Cancer Ther 9: 336-346, 2010.

70. Singh A, Boldin-Adamsky S, Thimmulappa RK, Rath SK, Ashush H, Coulter J, Blackford A, Goodman SN, Bunz F, Watson WH, et al: RNAi-mediated silencing of nuclear factor erythroid-2-related factor 2 gene expression in non-small cell lung cancer inhibits tumor growth and increases efficacy of chemotherapy. Cancer Res 68: 7975-7984, 2008.

71. Tian Y, Liu Q, He X, Yuan X, Chen Y, Chu Q and Wu K: Emerging roles of Nrf2 signal in non-small cell lung cancer. J Hematol Oncol 9: 14, 2016.

72. Qian Z, Zhou T, Gurguis CI, Xu X, Wen Q, Lv J, Fang F, Hecker L, Cress AE, Natarajan V, et al: Nuclear factor, erythroid 2-like 2 -associated molecular signature predicts lung cancer survival. Sci Rep 5: 16889, 2015.

73. Mahaffey CM, Zhang H, Rinna A, Holland W, Mack PC and Forman HJ: Multidrug-resistant protein-3 gene regulation by the transcription factor Nrf2 in human bronchial epithelial and nonsmall-cell lung carcinoma. Free Radic Biol Med 46: 1650-1657, 2009.

74. Liu X, Sun C, Liu B, Jin X, Li P, Zheng X, Zhao T, Li F and Li Q: Genistein mediates the selective radiosensitizing effect in NSCLC A549 cells via inhibiting methylation of the keapl gene promoter region. Oncotarget 7: 27267-27279, 2016.

75. Cho JM, Manandhar S, Lee HR, Park HM and Kwak MK: Role of the Nrf2-antioxidant system in cytotoxicity mediated by anticancer cisplatin: Implication to cancer cell resistance. Cancer Lett 260: 96-108, 2008

76. Copple IM, Dinkova-Kostova AT, Kensler TW, Liby KT and Wigley WC: NRF2 as an emerging therapeutic target. Oxid Med Cell Longev 2017: 8165458, 2017.

77. Magesh S, Chen Y and Hu L: Small molecule modulators of Keap1-Nrf2-ARE pathway as potential preventive and therapeutic agents. Med Res Rev 32: 687-726, 2012.

78. Abed DA, Goldstein M, Albanyan H, Jin H and Hu L: Discovery of direct inhibitors of Keap1-Nrf2 protein-protein interaction as potential therapeutic and preventive agents. Acta Pharm Sin B 5: 285-299, 2015.

79. Harder B, Tian W, La Clair JJ, Tan AC, Ooi A, Chapman E and Zhang DD: Brusatol overcomes chemoresistance through inhibition of protein translation. Mol Carcinog 56: 1493-1500, 2017.

80. Ren D, Villeneuve NF, Jiang T, Wu T, Lau A, Toppin HA and Zhang DD: Brusatol enhances the efficacy of chemotherapy by inhibiting the Nrf2-mediated defense mechanism. Proc Natl Acad Sci USA 108: 1433-1438, 2011.

81. Olayanju A, Copple IM, Bryan HK, Edge GT, Sison RL, Wong MW, Lai ZQ, Lin ZX, Dunn K, Sanderson CM, et al: Brusatol provokes a rapid and transient inhibition of $\mathrm{Nrf} 2$ signaling and sensitizes mammalian cells to chemical toxicityimplications for therapeutic targeting of Nrf2. Free Radic Biol Med 78: 202-212, 2015.

82. Kitamura $\mathrm{H}$ and Motohashi $\mathrm{H}$ : NRF2 addiction in cancer cells. Cancer Sci 109: 900-911, 2018 